\title{
Trigger Rate Dependence and Gas Mixture of MRPC for the LEPS2 Experiment at SPring-8
}

\author{
Hsieh Chia-Yu ${ }^{1}$ \\ Institite of Physics, Academia Sinica \\ 128 Sec. 2, Academia Rd., Nankang, Taipei 11529, Taiwan \\ cyhsieh0531@gmail.com
}

\section{Natsuki Tomida}

Division of Physics and Astronomy, Graduate School, Kyoto University

Kitashirakawa-Oiwakecho,Sakyo-ku,Kyoto606-8502,Japan

natsuki@scphys.kyoto-u.ac.jp

\section{Hiroaki Ohnishi}

RIKEN (The Institute of Physical and Chemical Research), Saitama, Japan

\section{Masayuki Niiyama}

Division of Physics and Astronomy, Graduate School, Kyoto University, Kyoto, Japan

\section{Chang Wen-Chen}

Institite of Physics, Academia Sinica, Taiwan

\section{Lin Willis Tsung-Tai}

Department of Physics, National Central University, Taiwan

In order to separate $\mathrm{K}$ and $\pi$ with momenta up to $1.1 \mathrm{GeV} / \mathrm{c}$ using Time-of-Flight (TOF) over a distance of about $100 \mathrm{~cm}$ in the magnet, we will use Multi-Gap Resistive Plate Chamber (MRPC) due to the need of large area, high efficiency and overall time resolution in the range of 50-100 ps. We built a $260 \mu \mathrm{m} \times 10$ gaps MRPC with an active area of $10 \times 50 \mathrm{~cm}^{2}$ and a pad size of $2.5 \times 40 \mathrm{~cm}^{2}$. We studied the trigger rate dependence and the gas mixture of this MRPC. It was found that high enough voltage is necessary to achieve a good time resolution and high efficiency at large trigger rates. The gas mixture composed of R134a : SF6 : Iso-Butane at 90\%:5\%:5\% is our optimized choice.

XI workshop on Resistive Plate Chambers and Related Detectors (RPC2012)

INFN-Laboratori Nazionali di Frascati, Italy

February 5-10, 2012

1 Speaker 


\section{Introduction}

A Resistive Plate Chamber (RPC) is composed by a gas volume enclosed by two resistive plates. High voltage is applied to the electrodes on the outer surface of these resistive plates, producing a high electric field across the gas gap. Ions and electrons are liberated in the gap by through-going charged particles. The avalanche forms when electrons and ions travel in the high electric field, thus inducing signals on the external electrodes. The electric field only decreases in proximity of the avalanche and the sensitivity out of this area remains unaffected. The Multigap Resistive Plate Chamber (MRPC) has more than one gap between the electrodes through the insertion of additional resistive plates. The time jitter of the chamber depends on the position of the primary ionization clusters in the gap. A wider gap leads to a larger time jitter (worse time resolution). MRPC can achieve a better time resolution with respect to RPC thanks to the fact that the single gap is smaller $[1,2]$. In recent years the MRPC has become a favourite choice for time-of-flight (TOF) systems in modern nuclear and particle physics experiments due to its easiness of construction, low cost, high efficiency and good time resolution.

\section{LEPS2 experiment at SPring-8}

The second generation Laser-Electron-Photon facility at SPring-8 (LEPS2) experiment for studying the photo-production of vector mesons and hyperons is scheduled to start in 2013. The backward Compton photon (BCS photon) beam with energy up to $3 \mathrm{GeV}$ is produced by scattering of laser photons on $8 \mathrm{GeV}$ electrons inside SPring- 8 storage ring. The development of a $4 \pi$ detector and the construction of a solenoid magnet is currently underway $[3,4]$. In order to separate $\mathrm{K}$ and $\pi$ with momenta up to $1.1 \mathrm{GeV} / \mathrm{c}$ while traveling a distance about $100 \mathrm{~cm}$ in a magnet, we need a large-area, high-efficiency TOF detector with a time resolution better than 50 ps and an efficiency better than $99 \%$ at a rate about $1 \mathrm{~Hz} / \mathrm{cm}^{2}$. The TOF detector covers a $4 \pi$ angle in an area of about $5 \mathrm{~m}^{2}$. Due to the limited budget we need to reduce the electronics to about 1000 channels. This means that the size of read-out per channel has to be larger than 50 $\mathrm{cm}^{2}$. MRPC is chosen for this purpose in LEPS2. In order to optimize the best configuration, the geometry of pad/strip, the gap width, the number of gaps, the number of stacks, the gas mixture, the trigger rate dependence were investigated in the beam test done in year 2011. The results on gas mixture and the trigger rate will be reported below while the other results are reported in [5].

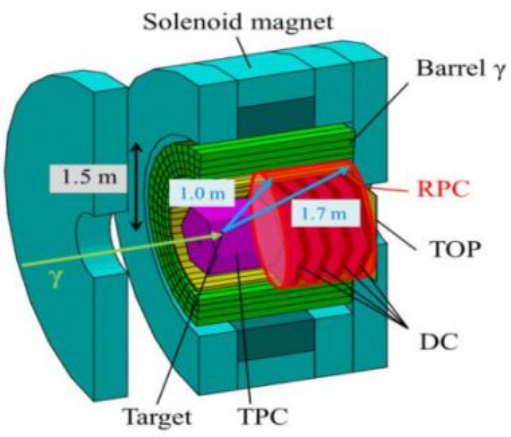

Figure 1. $4 \pi$ detectors of LEPS2 


\section{Description of the prototype MRPC}

We built a double-stack MRPC with five gaps for each stack and with a $260 \mu$ m gap width (260 $\mu \mathrm{m} \times 2$ stacks $\times 5$ gaps). There are two kinds of read-out shape: pad and strip read-out. Signals of pad read-out with an area of $1.5 \times 5.5 \mathrm{~cm}^{2}$ were taken from one end. Strips with an area of $2.5 \times 20 \mathrm{~cm}^{2}, 2.5 \times 40 \mathrm{~cm}^{2}, 1.5 \times 20 \mathrm{~cm}^{2}$ were read out from both ends. A sketch of the prototype MRPC with electrodes made of glass is shown in Fig. 2. The size of the outer glass is $100 \times 500 \mathrm{~mm}^{2}(0.5 \mathrm{~mm}$ thickness $)$ and that of the inner glass is $100 \times 500 \mathrm{~mm}^{3}(0.4 \mathrm{~mm}$ thickness). A fishing line is stretched across the surface of the glass and around the plastic screws along the edges as shown in Fig. 3. The fishing line acts as spacer between glass plates and creates the gas gap with a width equal to its diameter. The carbon tape on the surface of the outer glass was used to apply high voltage to the stacks. The resistivity of the carbon tape is $500 \Omega / \square$. The plastic bases are taped on the other side of carbon tapes. The pad and strip read-outs made by copper tapes were taped on the other side of plastic base.

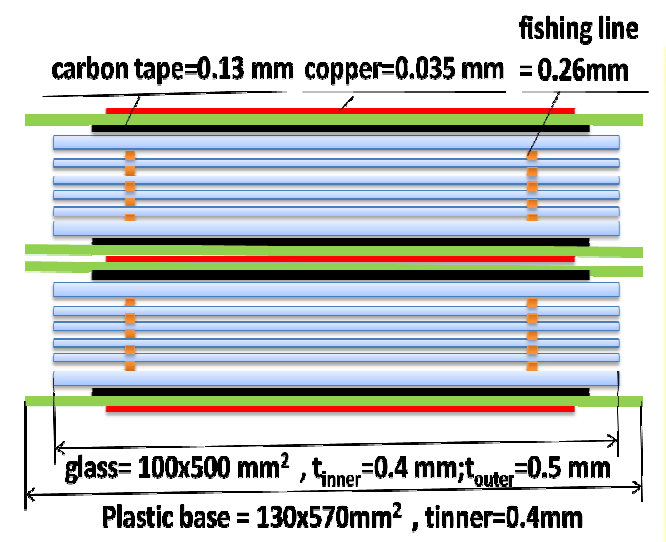

Figure 2. Sketch of prototype MRPC.
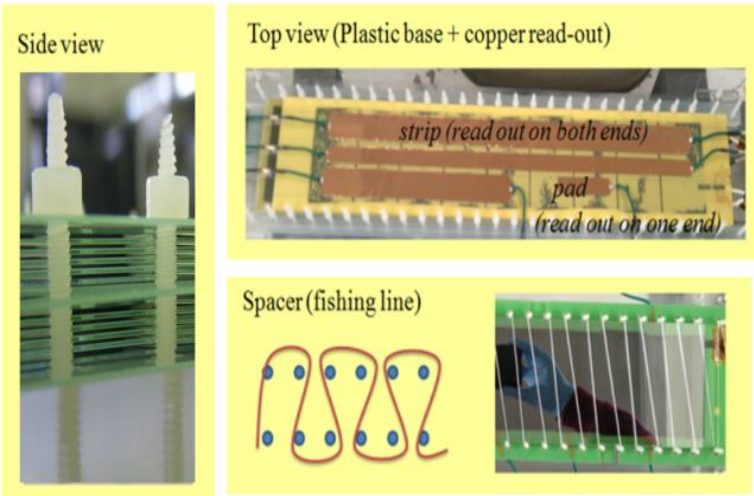

Figure 3. Photos of prototype MRPC.

\section{Beam test}

We have tested the prototype MRPCs in BL33 (LEPS's beamline) at Spring- 8 . The setup of the beam test with the chamber is shown in Fig. 4. The BCS photon beam collides with a lead converter $(\mathrm{Pb})$ and produces electron and positron pairs. Using a dipole magnet, we select electrons for our use. The MRPC, which is placed in the between four scintillators (S1-S4), measured the TOF of the electrons bended by the magnet. The trigger of the MRPC was defined as the coincidence of the four scintillators with a $1 \times 2 \mathrm{~cm}^{2}$ area. The trigger rate can be adjusted by the intensity of the laser. The analog signals were read out with a PMT amplifier module (KN2104) made by Japanese company Kaizu Work Corporation. After amplification the signals were split by a Fan-Out for charge and timing measurements respectively. Charge $Q$ was measured by a REPIC RPC-022 ADC. The TOF was measured by a GNC-040 TDC produced by the Japanese company Gnomes Design. 

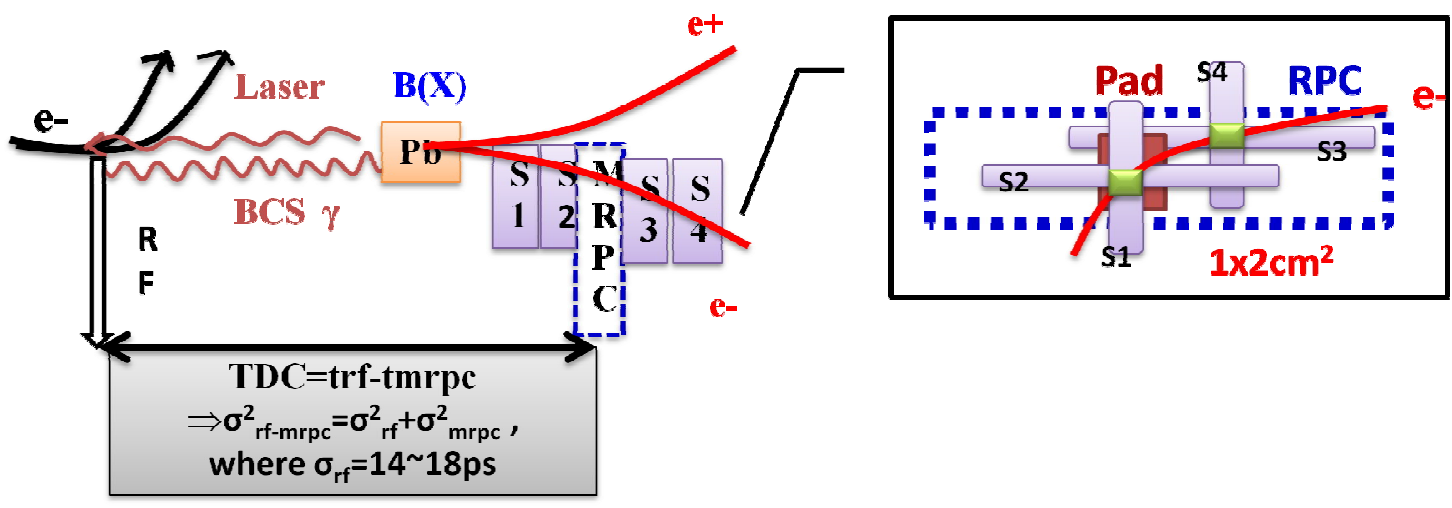

Figure 4. Sketch of the experimental set-up.

\section{Data analysis}

The TOF of the electrons between the converter and the MRPC was measured by a TDC. There are two important properties of the MRPC to be determined:

$>$ In the beam test the MRPC was in the center of four scintillators, S1-S4. The trigger of the MRPC was defined as the coincidence of the four scintillators with a $1 \times 2 \mathrm{~cm}^{2}$ area. Efficiency defined as the ratio between the number of the valid hits observed in the MRPC and the total number of the events.

$>$ The time resolution:

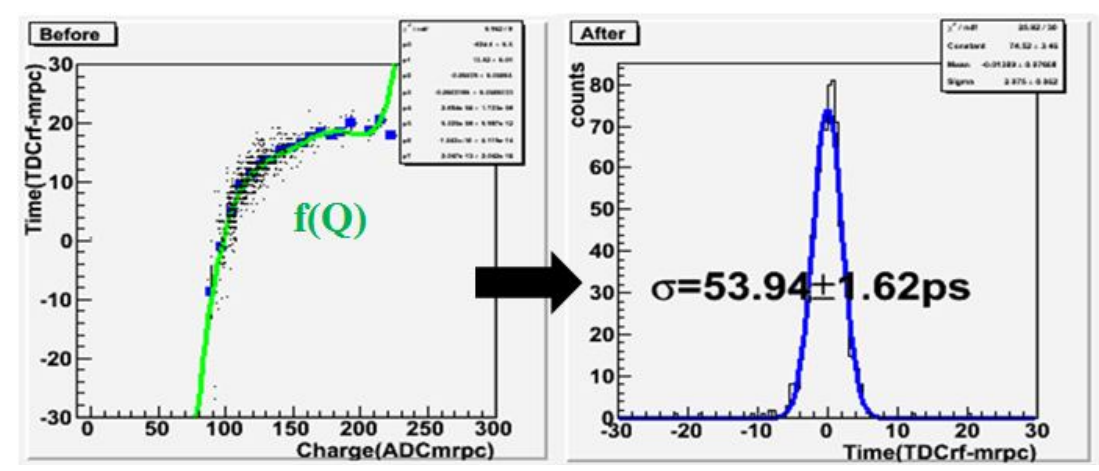

Figure 5. Slewing Correction.

The start signals for the TDC were provided by RF signals from the storage ring; stop signals were given by the MRPC. The time resolution receives a contribution by both RF and the MRPC. The time resolution of the RF signals is $14-18$ ps which is negligible compared to overall time resolution. Due to the time delay of the small signals, a slewing correction had to be applied according to the pulse-height spectrum. A plot of time vs. charge is shown in Fig. 5, together with a corresponding sixth-degree polynomial fit. The time resolution on RF plus the MRPC is obtained by the width of a Gaussian fit on the distribution of the corrected time. 


\section{Results and discussion}

The electron beam position was in the center of pad or strip read-out. For strip read-out the efficiency is defined as the coincidence and the time resolution is the mean time of ends.

\subsection{Tigger rate dependence}

The chamber was filled with gas mixture of $90 \%$ R134a, $5 \%$ SF6 and $5 \%$ Iso-butane. The performance of the MRPC with $2.5 \times 50 \mathrm{~cm}^{2}$ strip for three different trigger rates is shown in Fig. 6 in terms of efficiency, time resolution, average charge and time walk as a function of high voltage. The highest trigger rate is about $150 \mathrm{~Hz} / \mathrm{cm}^{2}$ due to the limitation introduced by the intensity of the laser. More than $98 \%$ efficiency is achieved for rates below $150 \mathrm{~Hz} / \mathrm{cm}^{2}$. With the increase of the rate, efficiency drops and time resolution also gets worse. A high enough voltage is necessary to achieve high efficiency and good time resolution for higher rate.
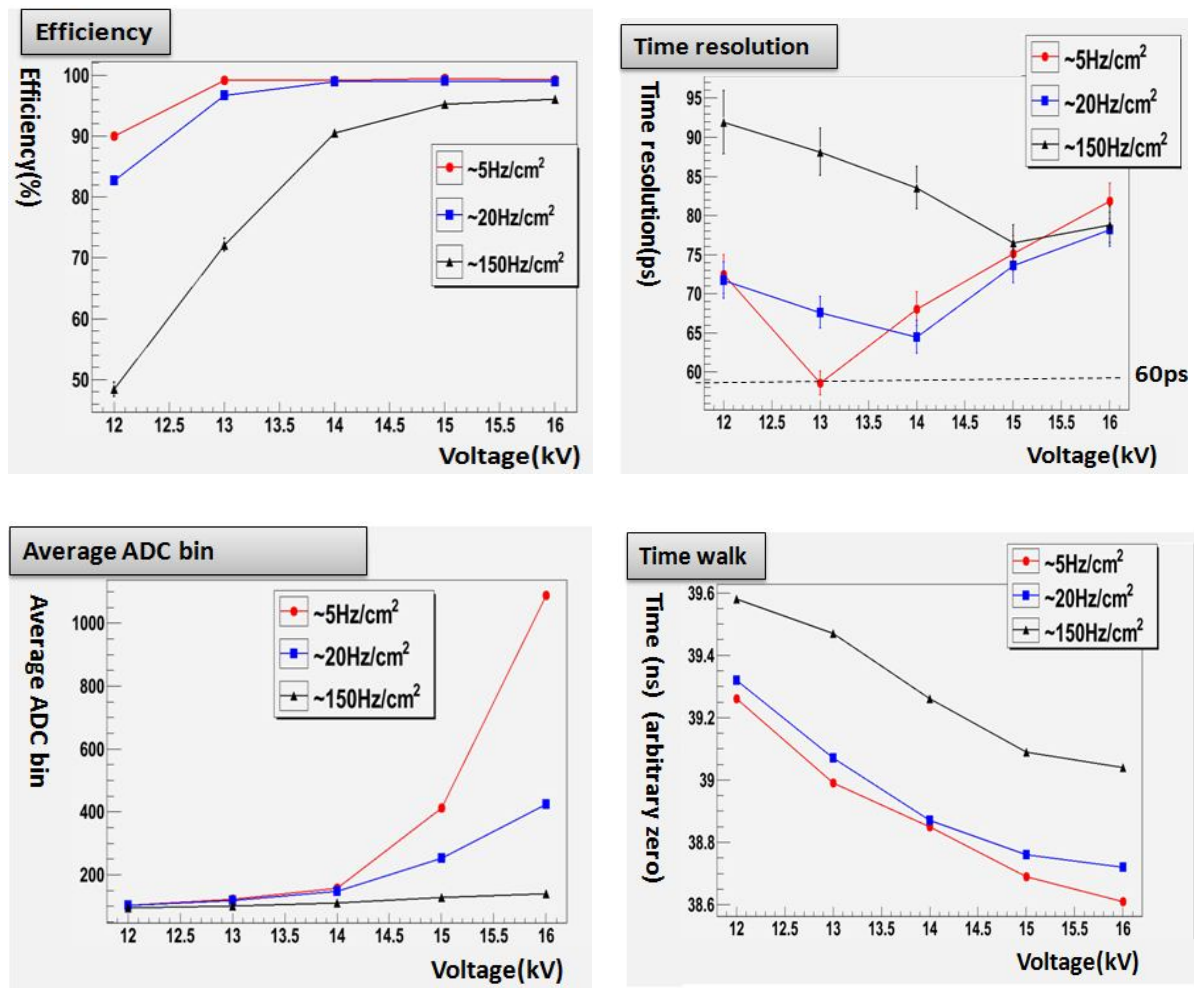

Figure 6. The behavior of the $260 \mu \mathrm{m} \times 2$ stacks $\times 5$ gaps MRPC with $2.5 \times 50 \mathrm{~cm}^{2}$ strip.

The chamber was filled with gas mixture of 90\% R134a, 5\% SF6 and 5\% Iso-butane.

The "Average ADC bin" variable represents the average charge of signals after amplifying about 16 times. Each count corresponds to $0.25 \mathrm{pC}$. The average charge is reduced at higher rates. Electrons and positive ions deposited on the surface of the plates reduce the electric field. This is the so called space-charge effect. The full field is later restored by the current flowing through the resistive plates. The current flowing into the glass increases when the trigger rate increases. This leads to a bigger voltage drop on the electrodes so that the induced 
signals are smaller. This explains why the high enough voltage is necessary to achieve high efficiency and good time resolution at higher rates. Because of the same reason, the drift velocity of electrons and ions became smaller. That's why the arriving time of signals is delayed at higher rates.

\subsection{Gas mixture}

Previously the gas mixture used for RPC chamber was at 90\% R134a, 5\% SF6 and 5\% Iso-butane. We replaced Iso-butane with Butane as a quencher. Theoretically Iso-butane is a better quencher than Butane because its structure is more symmetric and stable. On the other hand Butane is much cheaper than Iso-butane. A comparison, obtained using trigger rates, is shown in Fig. 7 obtained using a low trigger rate. The performance of Butane and Iso-butane is consistent in efficiency and time resolution at $13.5 \mathrm{kV}$. Butane shows a comparably good performance with respect to Iso-butane. The aging effect of Butane will be investigated in the future.
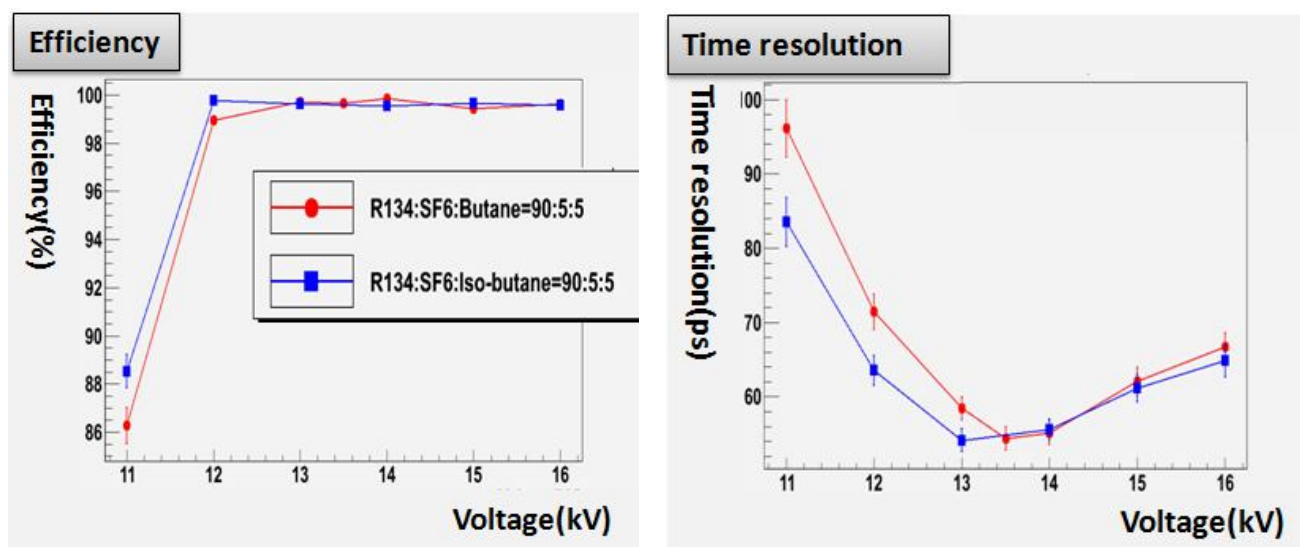

Figure 7. The behavior of the $260 \mu \mathrm{m} \times 2$ stacks $\times 5$ gaps MRPC with $1.5 \times 5.5 \mathrm{~cm}^{2}$ pad for gas mixture with Butane or Iso-butane.

\section{Conclusion}

A prototype MRPC $(260 \mu \mathrm{m} \times 2$ stacks $\times 5$ gaps $)$ with an area of $2.5 \times 40 \mathrm{~cm}^{2}$ strip has been tested with electron beam. An efficiency of $99 \%$ and time resolution of 60 ps have been achieved with a trigger rate below $1 \mathrm{~Hz} / \mathrm{cm}^{2}$. The gas mixture of the chamber was at $90 \% \mathrm{R} 134 \mathrm{a}$, $5 \%$ SF6 and 5\% Iso-butane. It takes about 1000 channels to cover the whole area of TOF detector. The performance reasonably meets the requirement for the LEPS2 experiment. In the future we will further study the effect of the impedance of the strip and develop the amplifier for the readout of the MRPC. Finally, a total area of $10 \times 100 \mathrm{~cm}^{2}$ MRPC will be built for the experiment.

\section{References}

[1] M.C.S. Williams, Nucl. Phys. B (Proc. Suppl.) 6IB (1998) 250-257 
[2] E. CerronZeballos et al. , Nucl. Instr. and Meth. A 367 (1995) 388-393

[3] M. Niiyama, Proceedings of Hadron2011, eConf C110613 (2011)

[4] M. Yosoi, AIP Conf. Proc. 1388, 163 (2011); doi: 10.1063/1.3647366

[5] M. Tomida, Proceedings of RPC2012, PoS(RPC2012)017 